\title{
Quand la technologie LiDAR révèle l'ampleur de cités enfouies
}

Damian Evans (damian.evans@efeo.net), Christophe Pottier et Dominique Soutif École française d'Extrême-Orient, 22 avenue du Président Wilson, 75116 Paris

De tout temps, nombre d'activités humaines ont durablement

transformé le paysage et, pour qui sait l'interpréter,

la topographie des sites constitue donc une archive précieuse des sociétés du passé.

La télédétection par LiDAR a récemment révolutionné cette approche en permettant d'analyser en détail la topographie de sites de grande ampleur, même dans des zones couvertes par une végétation dense.

Cet article prend pour exemple l'application récente de cette technique à l'exploration du complexe urbain d'Angkor, au Cambodge.

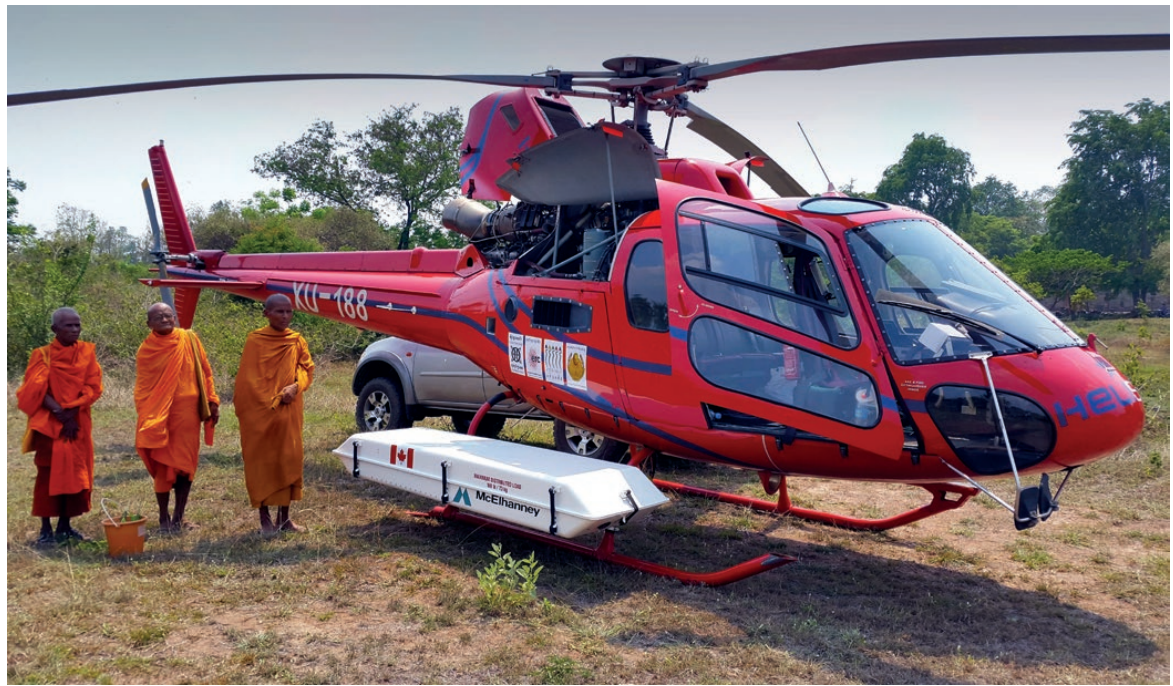

Trois bonzes de la pagode de Banteay Chhmar (au nord-ouest du Cambodge) préparent en 2015 une cérémonie pour bénir un nouvel instrument LiDAR.

\section{Le passé vu du ciel}

Dès le XIX ${ }^{\mathrm{e}}$ siècle, explorateurs et spécialistes des antiquités se sont efforcés de documenter le paysage des lieux qu'ils visitaient, rassemblant ainsi des indices en particulier, des vestiges architecturaux en brique et en pierre - qui permirent d'identifier nombre de sites depuis longtemps désertés, enfouis ou simplement masqués par la végétation. Au cours du $\mathrm{XX}^{\mathrm{e}}$ siècle, cette démarche a été progressivement stimulée, tant par des innovations technologiques que par l'évolution de l'archéologie en tant que discipline scientifique.

L'histoire des missions archéologiques françaises en Asie du Sud-Est, en cours depuis la fin du XIX ${ }^{\mathrm{e}}$ siècle, illustre bien l'évolution de l'archéologie du paysage et en souligne certaines limites, en particulier en milieu tropical. En effet, la végétation dense caractéristique de ce type d'environnement a longtemps rendu le relief difficile à appréhender, tant au sol que vu du ciel. Pourtant, des avancées significatives ont été très tôt réalisées, notamment par les chercheurs de l'École française d'Extrême-Orient (EFEO). Au Cambodge par exemple, des prospections archéologiques aériennes furent entreprises dès la fin des années 1920 par des aviateurs et archéologues qui palliaient l'absence de piste par l'emploi d'hydravions profitant des douves encore en eau autour des temples.

Angkor, qui fut la capitale de l'empire khmer du VIII ${ }^{\mathrm{e}}$ au $\mathrm{XV}^{\mathrm{e}}$ siècle, doit évidemment sa célébrité à ses grands sanctuaires - à commencer par Angkor Vat - qui ont longtemps monopolisé l'attention des chercheurs. Pourtant, en dehors des temples de pierres ou de briques, demeures des dieux, et de quelques ouvrages civils massifs, cette civilisation était par excellence "végétale». Du plus humble des serviteurs jusqu'au souverain, les mortels édifiaient en effet leurs bâtiments en matériaux légers aujourd'hui disparus. En surface, même érodés, seuls 
$>>$

les tertres, digues, canaux et bassins témoignent aujourd'hui de ces aménagements. Mais pendant longtemps, la densité de la couverture végétale rendait les prospections sur le terrain difficiles, et nous n'avions donc qu'une vision très partielle des implantations humaines de ce qui constituait pourtant l'une des régions les plus densément peuplées de cette époque [1].

\section{Paysages et lasers}

Dans les années 1990, alors que la péninsule indochinoise sortait enfin de décennies de conflits et que la paix ouvrait au Cambodge des opportunités uniques, les archéologues se tournèrent à nouveau vers l'imagerie aérienne pour préciser la cartographie archéologique. Outre son intérêt pour la gestion de sites face à un développement rapide du tourisme, celle-ci constituait un outil indispensable pour l'étude du territoire à l'heure où reprenait le débat sur la théorie qui proposait de comprendre Angkor comme une vaste "cité hydraulique" dont l'ascension et le déclin étaient intimement liés à la gestion de l'eau. Malheureusement, les limites de la télédétection et les difficultés des prospections sous la forêt tropicale laissaient dans nos cartes archéologiques d'Angkor d'importantes lacunes, en particulier dans son cœur monumental densément boisé.

Depuis une dizaine d'années, l'essor rapide du LiDAR (voir l'encadré, p. 67), technologie qui s'était lentement développée depuis les années soixante, a permis de résoudre ce problème. Les instruments de balayage laser aéroportés offrent aujourd'hui la capacité de voir «à travers " la couverture végétale. Le principe est simple : des millions d'impulsions laser sont envoyées vers le sol, la mesure du temps de retour de chaque signal permettant alors de localiser précisément le point de réflexion dans un espace tridimensionnel. Même si les impulsions sont en grande majorité réfléchies par la végétation, le faible pourcentage qui passe dans les interstices des strates végétales et parvient au sol suffit pour créer des modèles remarquablement détaillés du terrain dénudé de la forêt. Il fournit, en quelques heures, de vastes levés topographiques que des décennies d'arpentage n'auraient pu prétendre offrir avec une telle précision.

\section{Des complexes urbains révélés}

En 2012, l'EFEO a rejoint un consortium composé de sept partenaires pour entreprendre le premier programme LiDAR archéologique en Asie, couvrant plusieurs centaines de kilomètres carrés du nord du Cambodge [2]. Cette expérience à grande échelle a constitué une étape importante dans l'histoire de la télédétection et a déclenché une forte émulation dans le monde archéologique, y compris au Cambodge même, où une seconde campagne d'acquisition porte aujourd'hui la couverture à plus de 2000 kilomètres carrés [3]. Au-delà de la seule période angkorienne, ce sont plusieurs millénaires d'occupation humaine que ces données ont documentés. Elles nous fournissent de nouvelles clés pour suivre l'émergence et le développement des sociétés complexes qui s'épanouirent dans cette région, pour générer des modèles sophistiqués sur l'anthropisation du paysage et pour tester des hypothèses sur le déclin des royaumes et des empires.

À ce jour, les lasers nous ont montré comment les zones monumentales n'étaient pas seulement des centres sacrés et cérémoniels, mais étaient en fait des zones denses et formellement planifiées au sein de complexes urbains de plus faible densité qui s'étendaient entre les temples bien connus et bien au-delà (fig. 1). Ces zones ne ressemblent pas à des "villes " au sens conventionnel, mais sont indéniablement urbaines, ce qui nous invite à repenser la définition même de l'urbanisme dans le passé. Presque invariablement, les populations qui ont construit des villes dans cet environnement tropical difficile l'ont fait d'une manière qui a profondément transformé le paysage naturel, souvent au détriment de leurs sociétés sur le long terme. Le LiDAR révèle en effet des transformations à l'échelle régionale, dans lesquelles de vastes zones forestières ont été converties en zones urbaines et agricoles. Avec des rivières entières détournées et des infrastructures hydrauliques de vaste ampleur qui bouleversaient l'environnement, les projets d'ingénierie massifs étaient difficiles à maintenir à terme et, en raison de leur durabilité, ils créaient une sorte d'inertie matérielle qui minait la capacité de ces civilisations à s'adapter aux changements sociaux et environnementaux.

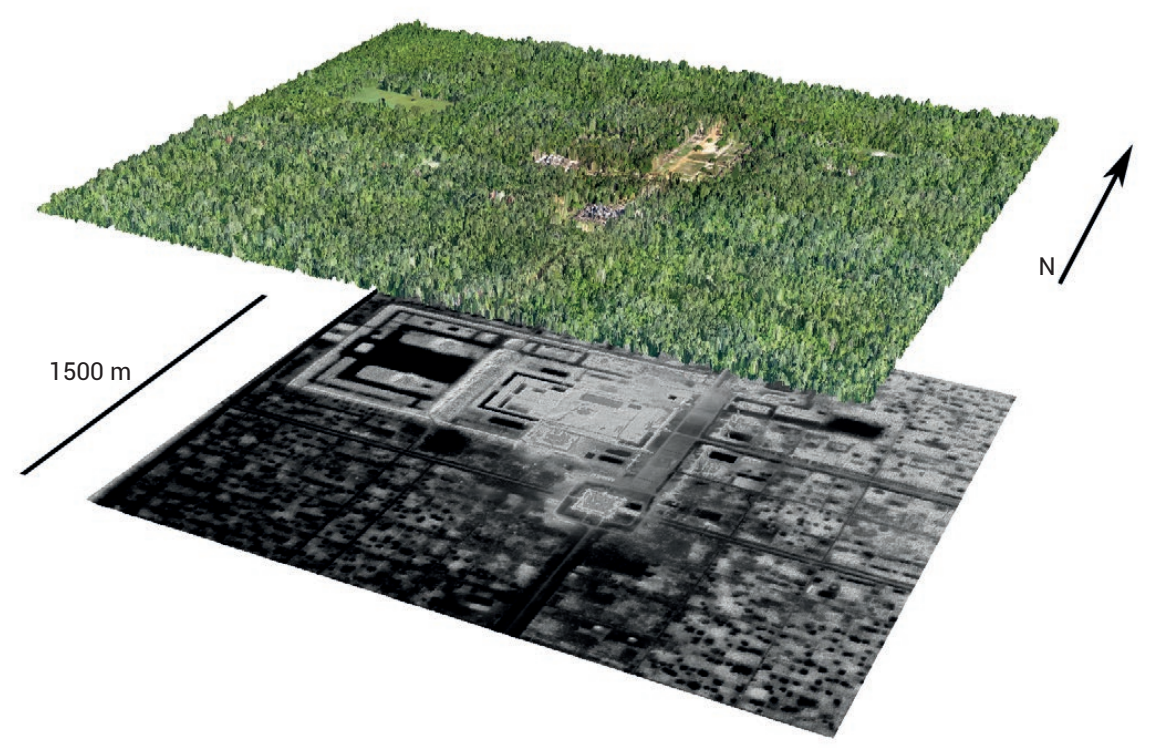

1. Vue oblique de la cité royale Angkor Thom. Le Bayon, le temple qui marque le centre de la ville d'Angkor Thom, est aujourd'hui enveloppé par la forêt (en haut). Les données LiDAR donnent à voir le réseau urbain qui l'entourait (en bas), ainsi que l'enceinte du palais royal (au nord-ouest), dont l'essentiel des infrastructures étaient construites en bois, et ont disparu depuis longtemps. 


\section{Principe de la télédétection par laser}

La télédétection par laser (ou LiDAR) est utilisée depuis les années 1960, mais ce n'est que récemment que cette technologie est devenue suffisamment sophistiquée et abordable par les archéologues pour un usage de cartographie sur des secteurs étendus. Les principes de base du balayage laser aérien sont en réalité plutôt simples : l'instrument est attaché à un engin volant, un hélicoptère par exemple (voir la photo, p. 65), et émet une pulsation laser infrarouge (1064 nanomètres dans notre cas) qui peut être réfléchie par une surface ou un " point d'impact " au sol, puis remonte au capteur ; ce dernier mesure et enregistre la durée de cet aller-retour, qui dépend bien entendu de la distance parcourue par la lumière. Ainsi, le capteur est informé de la distance le séparant du point d'impact, et l'engin volant étant lui-même équipé d'un GPS et d'une centrale à inertie qui enregistrent avec précision la position et l'orientation de l'équipement, nous pouvons finalement localiser avec une grande exactitude le point d'impact dans un système de coordonnées géographiques en trois dimensions.

La pulsation lumineuse ne pénètre pas vraiment la végétation, elle ne « voit " pas à travers ; en réalité, la lumière sera réfléchie par n'importe quelle surface et, pour les relevés en forêts tropicales, la majeure partie des retours laser sera issue de feuilles. Toutefois, un petit pourcentage des pulsations laser - en règle générale moins de $5 \%$ dans notre étude - va parvenir à se frayer un chemin à travers les minuscules jours de la canopée pour atteindre le sol de la forêt avant de remonter au capteur. C'est la raison pour laquelle le but pour les archéologues est de couvrir le terrain d'autant de pulsations laser que possible, afin de maximiser les retours provenant du sol ; nous avons besoin d'une densité de points inhabituelle en comparaison des autres usages, en écologie forestière ou cartographie générale par exemple. Dans notre cas, nous volons lentement et de façon systématique à environ 1000 mètres au-dessus du terrain, des pulsations laser étant émises à un rythme de 120 à 500 kilohertz, et nous enregistrons ainsi des millions de mesures par tranches de quelques minutes, le long de bandes d'environ 800 mètres de large. Notre but est d'obtenir jusqu'à 16 retours de points d'impact au sol par mètre carré, les données étant absolument fiables à l'échelle centimétrique. De cette manière, nous couvrons des centaines de kilomètres carrés en seulement quelques heures de vol, suivies de plusieurs semaines de post-traitement pour harmoniser les retours du laser avec les données spatiales.

Les données obtenues consistent fondamentalement (dans notre cas) en milliards de séries de coordonnées de type $X, Y, Z$. Les capteurs sont par définition parfaitement aveugles à la nature de l'objet réfléchissant la lumière, c'est pourquoi nous utilisons des algorithmes permettant de classifier les points d'impacts en post-traitement. Ce qui intéresse tout particulièrement les archéologues, c'est la classification automatique des points d'impact en catégories « sol » et « non-sol », capacité plutôt standard des outils logiciels de cartographie actuels. Une fois isolés, les points d'impact au sol peuvent être utilisés pour créer des maquettes " sol nu ", représentatives de vues du terrain efficacement "débarrassé " de sa végétation, et nous pouvons exploiter les schémas lumineux pour créer des représentations de "topographie archéologique", qui sont ensuite interprétées par les archéologues (fig. 1). Un intérêt particulier est accordé aux fondations en terre d'habitations en bois disparues depuis longtemps, et aux vestiges subtils de routes, canaux, fossés et structures de contrôle de l'eau. La résolution finale de ces images est limitée par la densité des données afférentes aux points d'impact au sol, mais pour nos objectifs nous produisons des images d'une résolution de 20 centimètres qui nous permettent de distinguer des objets d'aussi petite taille que des blocs de pierre sur le sol de la forêt.

\section{Une « révolution scientifique » dans la compréhension du passé ?}

Les récents progrès réalisés dans le monde par la technologie LiDAR ont encouragé les médias, et même certains chercheurs, à annoncer une "révolution scientifique " en archéologie comparable à l'avènement de la datation par radiocarbone dans les années 1950 [4]. Dans ce contexte, il est important d'être prudent par rapport à la méthode et de reconnaitre avant tout qu'elle est ancrée dans une évolution des méthodes et des idées, remontant aux premiers explorateurs. Si, dans certaines régions, les paysages révélés étaient largement inconnus, dans la plupart des cas, le LiDAR vient surtout combler les lacunes de connaissances existantes, loin donc des récits racoleurs de "villes perdues " et de " civilisations oubliées ".

Ce qui est pourtant indéniable, c'est que presque partout où nous regardons avec cet instrument, il apparait que nous avions sous-estimé l'échelle et l'intensité de l'effort humain enregistré à la surface de la Terre. Les paysages que nous imaginions auparavant comme "sauvages" ou "inhospitaliers " se révèlent façonnés par la main humaine ; certains prétendent que même l'Amazonie, par exemple, ressemble davantage à un jardin qu'à un phénomène naturel [5]. Et seuls des outils comme le LiDAR permettent de prendre la mesure de sites archéologiques à une telle échelle.

Dans les années à venir, les progrès techniques dans les technologies des drones et du LiDAR devraient finalement se combiner au point où ce dernier deviendra un élément standard de la boîte à outils de l'archéologue. Des perspectives plus importantes suivront sûrement dans le monde entier.

\section{Références}

1 C. Pottier, «Angkor et ses cartes" dans Anamorphoses. Hommage à Jacques Dumarçay, pp. 427-442, Les Indes Savantes, Paris (2006).

2• D.H. Evans et al., PNAS 110 (2013) 12595-12600

3. D. Evans, J. of Archaeol. Sci. 74 (2016) 164-175.

4 A.F. Chase et al., PNAS 109 (2012) 12916-12921.

5 P. Roberts et al., Nature Plants 3 (2017) 17093 